\title{
Silicon Photonic Accurate Wavelength Detection using an AWG and Time-Domain Multiplexing
}

\author{
Alfonso Ruocco*, Dries Van Thourhout*, Wim Bogaerts*†, \\ ${ }^{*}$ Photonics Research Group, Ghent University - imec,Department of Information Technology \\ Center of Nano- and Biophotonics (NB-photonics), Ghent, Belgium \\ $\dagger_{n}$ now also with: Luceda Photonics, Dendermonde, Belgium \\ Email: Alfonso.Ruocco@intec.UGent.be
}

\begin{abstract}
We present a silicon-on-insulator (SOI) compact wavelength detector. An AWG in Vernier configuration is used as wavelength selective device and thermo-optic MZ modulators perform time-domain multiplexing on the inputs. The measured average accuracy is $180 \mathrm{pm}$.
\end{abstract}

\section{INTRODUCTION}

Many applications require accurate wavelength detection. Integrated silicon wavelength filters [1], [2] often don't provide the required accuracy, but this can be improved using more complex optical circuits. Silicon photonics offers a largescale integration platform for many optical functions, where the combined functionality can outperform individual building blocks. We present spectrometer for accurate wavelength detection using active multiplexing, combining TDM (Time Division Multiplexing) techniques with an Arrayed Waveguide Grating (AWG). The circuit consists of an array of thermooptic Mach-Zehnder modulators and an AWG, as shown in Fig.1. Using a Vernier configuration with 4 input channels and five output channels with an FSR of 20nm, the wavelength accuracy is increased from approximately $1 \mathrm{~nm}$ to $180 \mathrm{pm}$.

\section{DESCRIPTION OF THE CIRCUIT}

The photonic circuit consists of a single $4 \times 5$ AWG, and four thermo-optic MZI modulators, one for each input channel of the AWG. The optical circuit is realized in the passive SOI technology platform of IMEC [3]. Fig.1 shows the schematic and a microscope image of the circuit. The input light is split into the four branches with an MZI modulator that is operated as an on-off switch. The output is then fed into one of the $M=4$ AWG inputs. The AWG has $N=5$ outputs: the relation in between the number of channels and the channel spacing $\Delta \lambda$ is expressed by

$$
F S R=M \cdot \Delta \lambda_{\text {in }}=N \cdot \Delta \lambda_{\text {out }} \frac{M}{N}=n_{\text {vernier }}
$$

where $n_{\text {vernier }}$ is the Vernier factor. Fixing the FSR guarantees a cyclic behavior of our device. In our device the channels spacings are $\Delta \lambda_{i n}=5 \mathrm{~nm}$ and $\Delta \lambda_{\text {out }}=4 \mathrm{~nm}$ respectively for input and output, amounting to an FSR of $20 \mathrm{~nm}$. The multiple inputs in vernier configuration can extract much more spectral information from the same AWG if we can read out all the input-output transmissions. When we use all the inputs simultaneously, the respective intensities are added incoherently in the photodetectors at the output. Therefore we
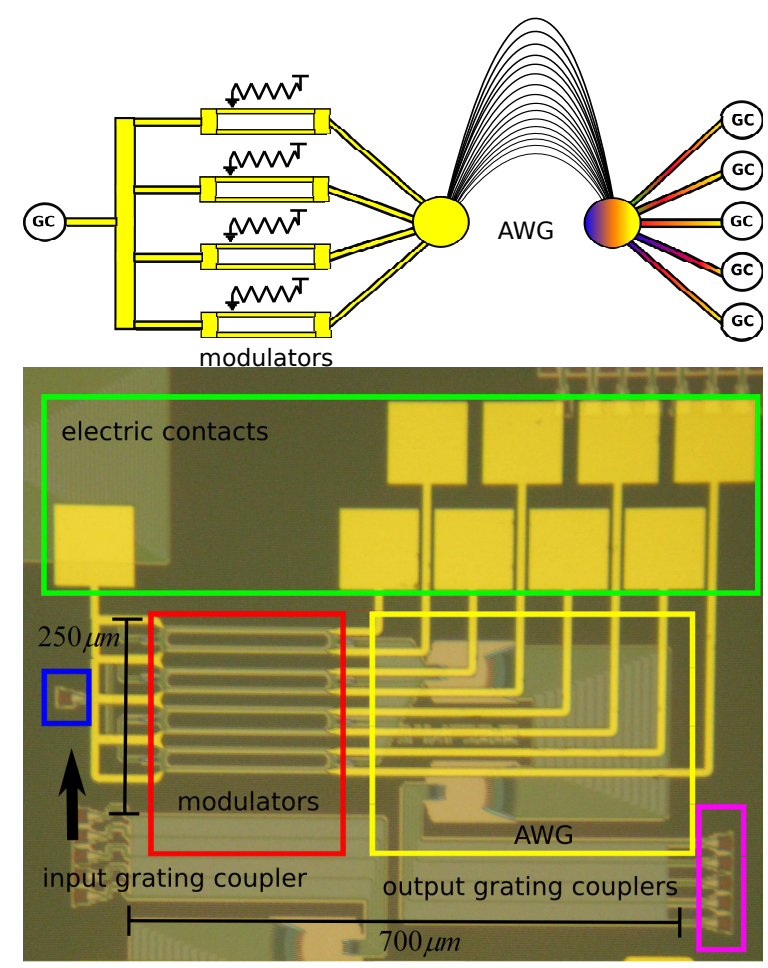

Fig. 1. Schematic and Microscope image of the spectrometer

label the input signals with a multiplexing technique. In this work, we use simple TDM, switching on a single channel at a time. We can then separate the different inputs with signal processing techniques. The active labeling is done with a bank of four thermo-optic MZI modulators: on top of the oxide cladding of the MZI waveguides, titanium heaters are processed. These heaters are capable of inducing a $\pi$ phase shift and modulating the output intensity between 0 and 1 .

\section{Simulation OF THE PIC}

We use the Caphe [4] circuit simulator is used to model the device. Each single sub-component is described by an s-matrix and the entire circuit is then simulated in the time domain. The AWG simulation is autmatically broken up into separate parts using the IPKISS design framework [5]. Fig. 2 shows the simulation of the device: the different sub-plots show the signals coming from each input already 'de-labeled'. 


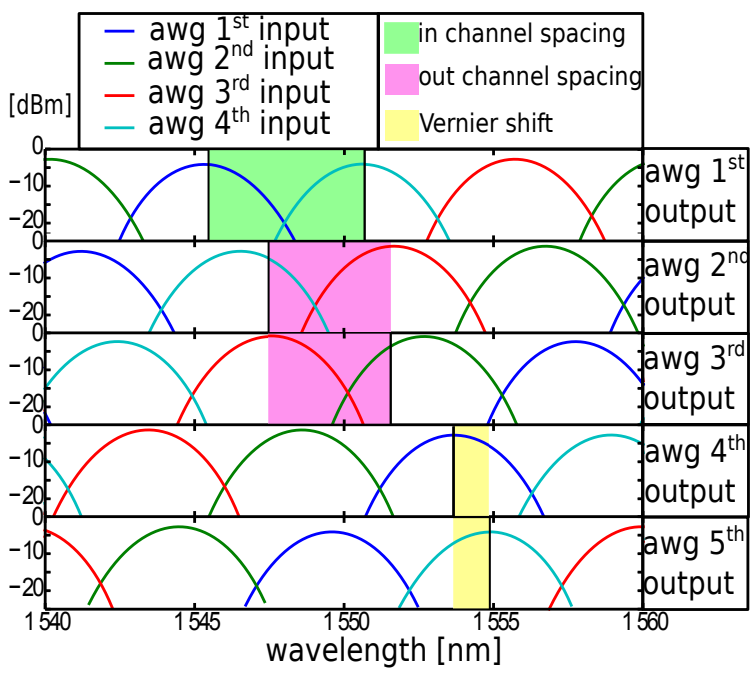

Fig. 2. Spectral simulation of the spectrometer

\section{EXPERIMENTAL RESULTS}

We apply a CoG (Center of Gravity) algorithm for the calculation of the peak position [6]. An equivalent mass assigned to each channel, scaled with the channel intensity. This weighing factor is higher when the measured wavelength peak is closer to the center of the channel. We can express the $\mathrm{CoG}$ algorithm as

$$
\lambda_{\text {meas }}=\frac{1}{\sum_{m=1}^{M} S_{n, m}} \sum_{m=1}^{M} \sum_{n=1}^{N} \lambda_{n, m} S_{n, m}
$$

with $S_{n, m}$ the AWG transmission of input $m$ to output $n$. The average accuracy of the measurement is evaluated as

$$
S_{a c c}=\frac{\sum_{n=1}^{M}\left(\sqrt{\left.\lambda_{i n}-\lambda_{\text {meas }}\right)^{2}}\right.}{M}
$$

While in a single-input AWG only two channels contribute to a wavelength measurement, in our configuration the Vernier effect makes sure than multiple channels can be used in the CoG method. As the noise floor can affect this algorithm, we apply a threshold, ignoring all contributions below a certain intensity. The thermo-optic switches are characterized separately to apply their own calibrated drive current.

Fig. 3 shows the result of the TDM measurement with all the input-to-output spectra disentangled. Due to fabrication variations, the center wavelength of the AWG is shifted, but this is not an issue as the device was designed with a cyclic response. Fig. 4 shows the measured accuracy over the $20 \mathrm{~nm}$ FSR. The average accuracy is $180 \mathrm{pm}$, according to eq. 3 .

\section{CONClusions}

In this paper we demonstrated a novel configuration of an active AWG-based spectrometer for peak detection implemented in silicon photonics. By using the Vernier effect and time-domain multiplexed inputs in our AWG, we can improve the peak detection resolution by a factor of 10 compared to

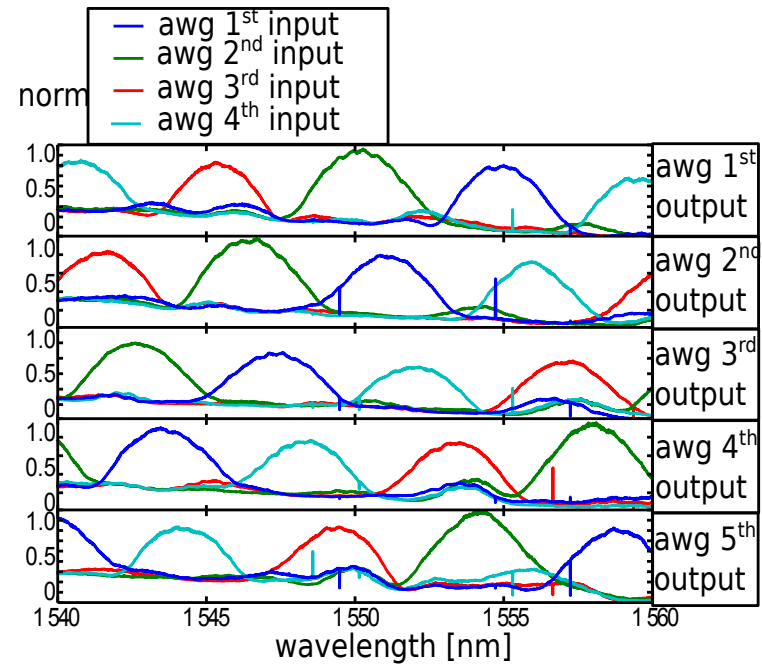

Fig. 3. Spectral measurements of the spectrometer

err $[\mathrm{nm}]$

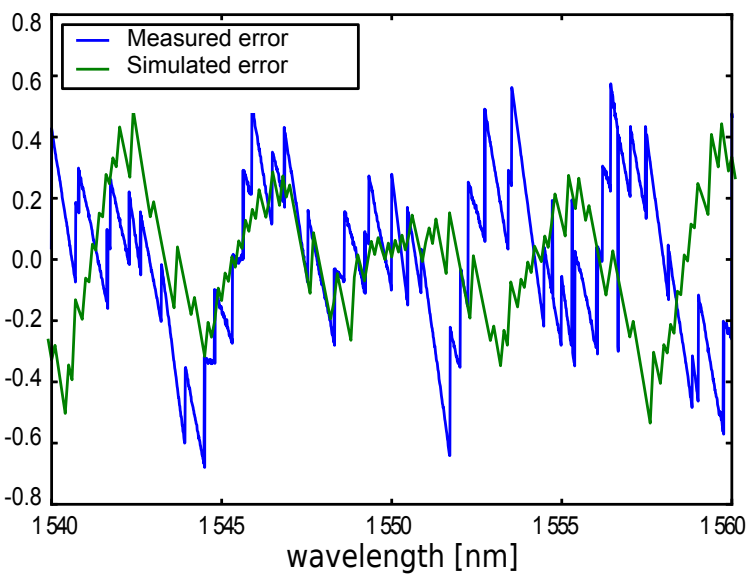

Fig. 4. Spectrometer local accuracy, simulated and measured

single input devices. The experimental performance demonstrated an average accuracy of $180 \mathrm{pm}$ with CoG algorithm across the FSR of the WDM device $(20 \mathrm{~nm})$. The concept can be scaled to a larger channel count for even higher resolutions.

\section{ACKNOWLEDGMENTS}

This work was supported by the EU FP7-SmartFiber project.

\section{REFERENCES}

[1] W. Bogaerts, S. Selvaraja, P. Dumon et al. J. Sel. Top. Quantum Electron., 16(1), pp. $33-44,2010$.

[2] J. Brouckaert, W. Bogaerts, S. Selvaraja et al. IEEE Photon. Technol. Lett. 20(4), pp. 309-311, 2008.

[3] S. Selvaraja, P. Jaenen, W. Bogaerts et al. J. Lightwave Technol. 27(18), pp. 4076-4083, 2009.

[4] M. Fiers, T. Van Vaerenbergh, K. Caluwaerts et al. J. Opt. Soc. Am. B 29(5), pp. 896-900, 2012.

[5] W. Bogaerts, Y. Li, S. Pathak et al. Proc. SPIE 8781, pp. 878-878, 2013.

[6] C. Askins, M. Putnam, and E. Friebele, in Smart Structures \& Materials 95. International Society for Optics and Photonics, 1995, pp. 257-266. 